\title{
COVID-19 model-based practice changes in managing a large prostate cancer practice: following the trends during a month-long ordeal
}

\author{
Kulthe Ramesh Seetharam Bhat ${ }^{1}$ (1) - Marcio Covas Moschovas ${ }^{1} \cdot$ Travis Rogers $^{1} \cdot$ Fikret F. Onol $^{1} \cdot$ Cathy Corder $^{1}$. \\ Shannon Roof ${ }^{1} \cdot$ Chiara Sighinolfi' $^{2} \cdot$ Bernardo Rocco $^{2} \cdot$ Vipul R. Patel $^{1}$
}

Received: 4 June 2020 / Accepted: 8 June 2020 / Published online: 14 June 2020

(c) Springer-Verlag London Ltd., part of Springer Nature 2020

\begin{abstract}
Coronavirus (COVID-19) has been a life-changing experience for both individuals and institutions. We describe changes in our practice based on real-time assessment of various national and international trends of COVID-19 and its effectiveness in the management of our resources. Initial risk assessment and peak resource requirement using the COVID-19 Hospital Impact Model for Epidemics (CHIME) and McKinsey models. Strengths, weaknesses, opportunities, and threats (SWOT) analysis of our practice's approach during the pandemic. Based on CHIME the community followed $60 \%$ social distancing, the number of expected new patients hospitalized at maximum surge would be 401 , with 100 patients requiring ventilator support. In contrast, when the community followed 15\% social distancing, the maximum surge of hospitalized new patients would be 1823 and 455 patients would require a ventilator. on April 15, the expected May requirement of ICU beds at peak would be 68 , with 61 patients needing ventilators. The estimated surge numbers improved throughout April, and on April 22 the expected ICU bed peak in May would be 11.7, and those requiring ventilator would be 10.5. Simultaneously, within a month, our surgical waitlist grew from 585 to over 723 patients. Our SWOT analysis revealed our internal strengths and inherent weakness, relevant to the pandemic. A graded and a guarded response to this type of situation is crucial in managing patients in a large practice.
\end{abstract}

Keywords COVID-19 SWOT analysis $\cdot$ Clinical practice $\cdot$ Practice management

\section{Introduction}

Coronavirus (COVID-19) has been a life-changing experience for both individuals and institutions. The recent global events involving COVID-19 have placed a major burden on the economy and more specifically the health care sector. Unfortunately, an increase in the volume of acute care patients, secondary to COVID-19, has resulted in an interruption of care for patients with chronic illness. Cancer is one example of a nonacute illness that has taken a backseat

Kulthe Ramesh Seetharam Bhat seetharam_bhat2003@yahoo.co.in; bhat.seetharam@gmail.com

1 Adventhealth Global Robotics Institute, Advent Health Celebration Health, Suite 200, 410 celebration place, Celebration, FL 34747, USA

2 Department of Urology, Ospedale Policlinico e Nuovo Ospedale Civile S. Agostino Estense Modena, University of Modena and Reggio Emilia, Modena, Italy
[1]. Considering that prostate cancer is thought to be a relatively stable cancer when compared to other urological malignancies, it is somewhat fortunate that our practice consists mainly of prostate cancer patients. However, in recent years, it became evident that a delay in recently due diagnosis can translate into an increased rate of aggressive cancer, as evident after the delay in diagnosis, thanks to the USPSTF recommendations against screening. Actually, after 2012 we have a more high risk (HR) and high-volume diseases being were referred to our surgical practice $[2,3]$. In response to this, we have had to deftly manage our practice so that we give preference to these patients. We adopted a risk-stratified approach in prioritizing patients while managing our practice. 


\section{Objective}

To describe changes in our practice based on real-time assessment of various national and international trends of COVID-19 and its effectiveness in the management of our resources.

\section{Materials and methods}

To determine the gravity of the COVID-19 situation, we performed an initial risk assessment and peak resource requirement using the COVID-19 Hospital Impact Model for Epidemics (CHIME), assuming 60\% and 15\% compliance in social distancing [4]. Using McKinsey models, we assessed the COVID-19 surge capacity requirement at different time intervals [5]. Trends in the severity of prostate cancer within our practice's patient population were identified weekly. These trends were assessed using the NCCN risk stratification system. Strengths, weaknesses, opportunities, and threat (SWOT) analysis of our practice's approach to the COVID-19 pandemic was also performed to help us effectively manage our resources [6].

\section{Results}

Close monitoring of the COVID-19 pandemic on both the national and international level led to our practice deciding to only operate on NCCN high-risk patients starting on March 15, which lasted until the end of March, when the operating rooms were closed to nonemergent cases by our hospital administrators.

\section{Trends of new cases in reference regions}

Figure 1 shows a comparison of trends of new cases within our reference regions of Italy and New York. Florida began its upward trend a week after New York state, which in turn had begun its upward trend 2 weeks after Italy. These trends were closely monitored by our risk management team and by us as a practice. The maximum number of cases in Italy was 6557 and the maximum number of cases in New York was 11,571 [7]. Though the overall number of cases was higher in New York, the number of deaths was higher in Italy.

\section{Initial risk assessment}

Based on our initial assessment using the CHIME tool, if the community followed $60 \%$ social distancing, the number of expected new patients hospitalized at maximum surge would be 401, with 100 patients requiring ventilator support. In contrast, when the community followed $15 \%$ social distancing, the maximum surge of hospitalized new patients would be 1823 and 455 patients would require a ventilator. The compiled data included all Advent health locations in central Florida (Fig. 2).

\section{The philosophy behind OR closure}

As the COVID-19 situation evolved, the hospital stockpile of personnel protective equipment (PPE) and the overall bed count was assessed. The results of the hospital PPE/ bed assessment, the initial CHIME model results, and the fact that COVID-19 cases were increasing on a national and international level (Fig. 1) led to our decision to suspend all surgical procedures. If we assume the need for 6 PPE units (each PPE unit would include headgear, masks, gowns, eye shields, a pair of gloves and foot covers) per prostatectomy, and knowing that we normally perform 8 prostatectomies a day, 4 days a week, our estimated PPE requirement would be 192 units a week and 768 units a month. Moreover, all of these patients would require a hospital bed for a minimum of 1 day, which would occupy eight beds for at least one night.

With concerns of a PPE shortage and an anticipated surge in the need for PPE, several policy changes were put in place. These included reusing PPE, especially N95 masks, work from home strategies for non-clinical staff, social distancing, and teleconsultation. The OR was closed on the 1st of April 2020, due to the COVID-19 situation and the potential burden on our hospital system.

\section{Monitoring the situation}

Various models were frequently developed by our risk management team and these were all analyzed by our administration (Fig. 3). In the worst-case scenario, a viral surge was expected in mid-May (Fig. 2). Throughout April, based on COVID-19 caseload and hospital resources, several McKinsey models were developed and used as a part of our risk mitigation strategy. These models were used to estimate the probable ICU census, including ventilated patients, and the total admission census in our center. The modeling varied based on the level of incidence, which was categorized into three different categories: baseline, low incidence, and high incidence. Based on these models, on April 15, the expected May requirement of ICU beds at peak would be 68 , with 61 patients needing ventilators. However, over time due to measures like "stay at home" orders by the governor and social distancing practices followed by the community, the estimated surge numbers improved throughout April, and on April 22 the expected ICU bed peak in May would be 11.7, and those requiring ventilator would be 10.5. The curves 
Fig. 1 Trends of new cases in coronavirus in Italy, New York state and Florida as of 30th Apr 2020

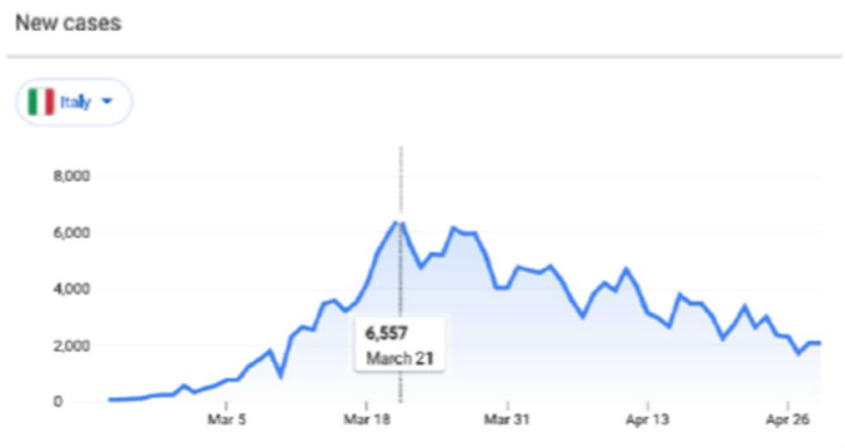

Total confirmed cases 204,000

Total deaths $-27,682$

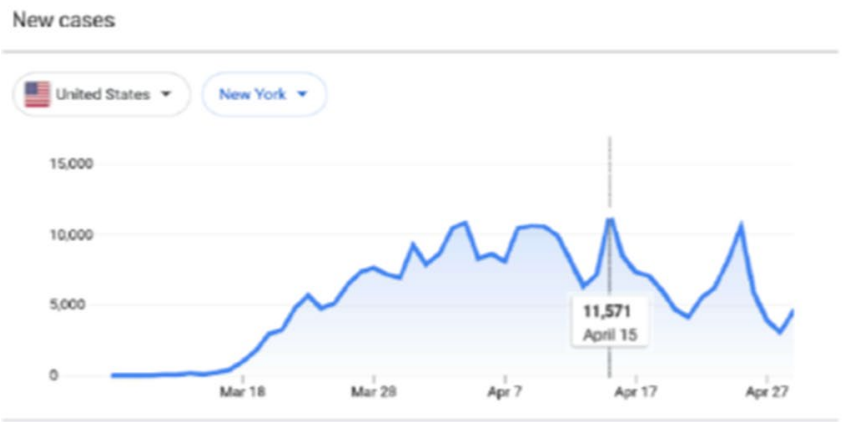

New cases

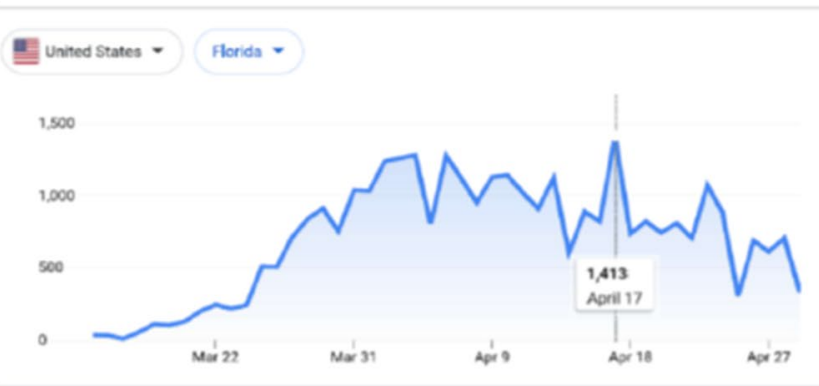

Total confirmed cases 300,000

Total deaths $-18,015$ remained stable on April 28, suggesting that mitigation strategies followed in Florida were ultimately effective.

The actual incidence of new cases at all AdventHealth locations in central Florida was tracked using laboratory data. The number of new cases diagnosed remained constant and started to decline in the last 4 days of April (Fig. 4).

\section{Assessment of hospital inventory}

Meanwhile, we assessed our hospital system's inventory for all the locations in central Florida. The overall available beds increased in response to the coronavirus pandemic. Over time, the ICU bed shortage reduced and the overall bed shortage was calculated to be negative at the end of April. These numbers were calculated assuming a $4 \%$ hospitalization rate. The initial shortage of beds, ICU beds, and ventilators needed, respectively, changed from 342, 706, and
199 to 481, 301, and 55, respectively (Fig. 5). The overall bed capacity was increased from 2993 to 3297.

\section{New patient management}

All new and existing patients had a one on one telehealth consultation, with a provider. These telehealth consultations were done instead of an office visit. The above-mentioned stratification protocol was used. The new patients had preoperative counseling via teleconsultation and were advised to follow precautionary social distancing guidelines established by the government, as well as, stay at home orders where applicable. They were advised to self-monitor for symptoms of COVID-19.

These patients were added to the surgical list following their teleconsultation. Every week, we stratified new patients to our practice based on the NCCN risk 
Fig. 2 Initial risk-assessment CHIME model using different levels of social distancing

\section{Presumed social distancing at $\mathbf{6 0 \%}$}
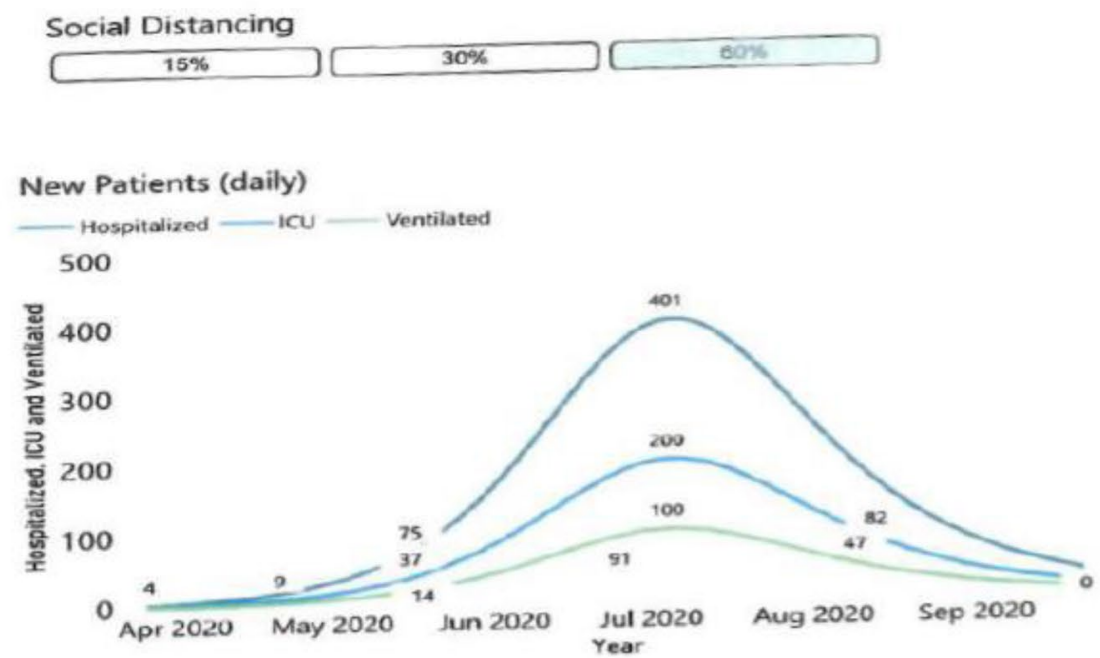

\section{Presumed social distancing at $15 \%$}
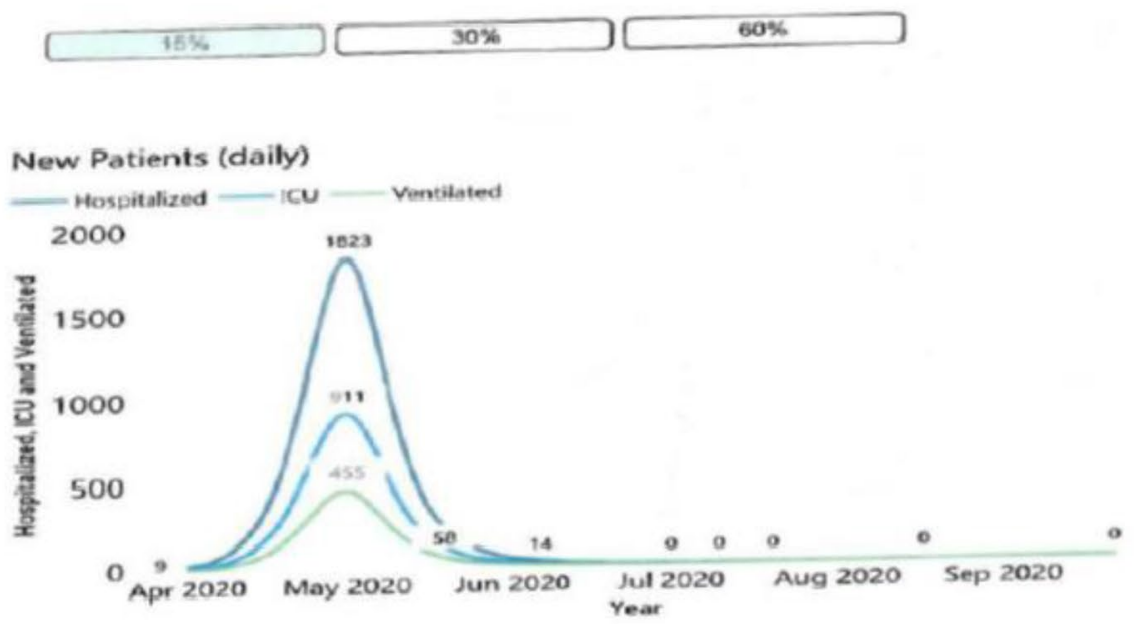

stratification. For scheduling, the HR and the very high risk (VHR) were categorized in one group to be prioritized for surgery, and the other group would include intermediate, low, and very low-risk groups. The trends in the number of cases on the waitlist are depicted in Fig. 6. Within a month, our surgical waitlist grew from 585 to over 723 patients, with an overall increase in every risk group. At the end of April, we had a total of 723 prostate cancer patients on our waitlist, with 216 of those being categorized as high and very high risk.

\section{Managing surgical waitlist}

As of April 1, 2020, we had 585 patients waitlisted for surgery, and these patients were categorized into groups based on the NCCN risk stratification. In the beginning, we had 45 VHR patients and $145 \mathrm{HR}$ as per NCCN risk categories. These patients were being prepared for surgery during the temporary shutdown. They were then assessed using COVID-19 risk scores to identify patients who were suitable for surgery (COVID-19 comorbidity risk less than 5), and these were selected to our first group of patients who would have surgery. 


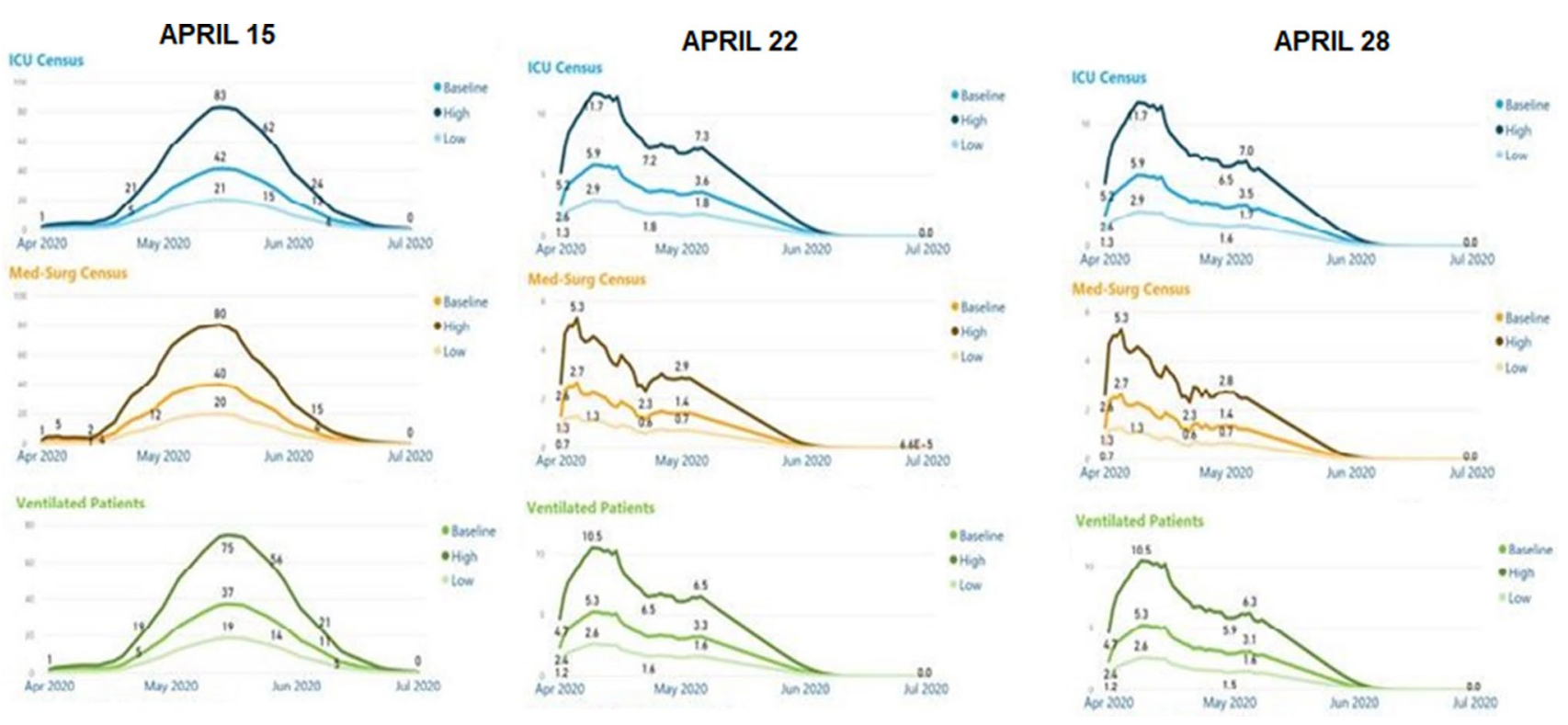

Fig. 3 McKinsey model COVID surge capacity assessment tool at different time period based on existing admission statistics

Fig. 4 Trends in new COVID19 positive cases at all Adventhealth location in Central Florida

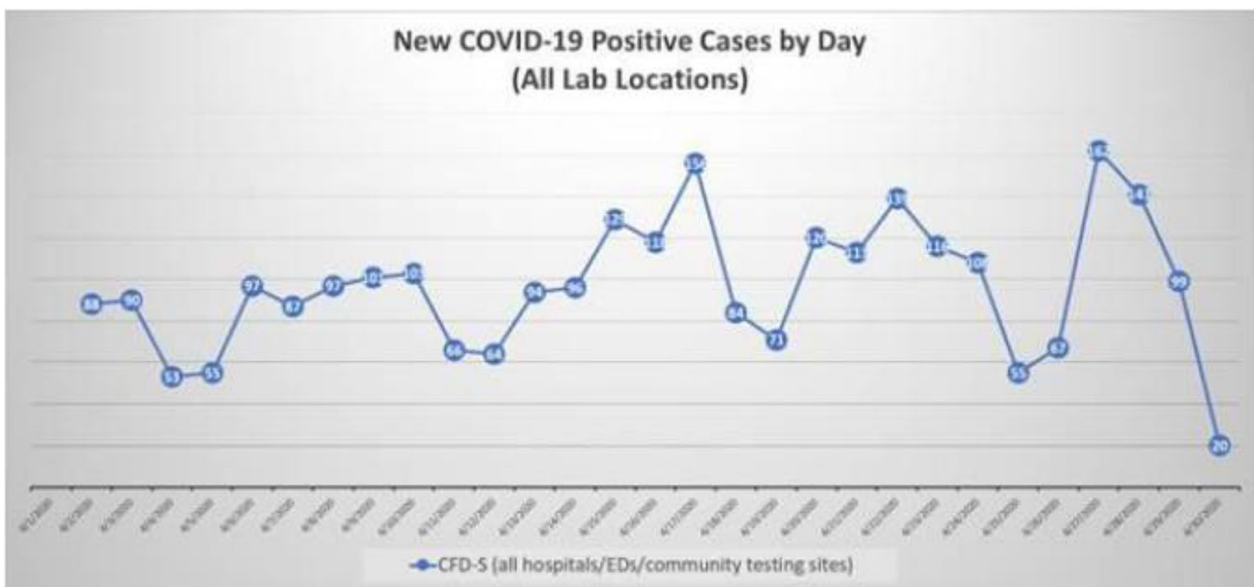

This was a recommendation that was based on a multinational DELPHI consensus that combined the risk score by COVID19 risk by MD Calc.com, Charlson co-morbidity index, and common sense (unpublished) (Table 1). All the VHR and HR patients were put on neoadjuvant ADT [8]. We deferred surgery for all the intermediate and low-risk patients until the COVID-19 pandemic was controlled to a safe level. Also, the patients in the COVID-19 high-risk group had their surgery deferred due to the possible risk of increased mortality and complication rates in patients undergoing surgery in the middle of the pandemic.

\section{SWOT analysis of our approach to this situation}

\section{Strengths}

The major strength of our approach was that it was a graded approach with modifications based on current evidence. This temporary shutdown helped us as a team to reassess our strategy of patient scheduling. We now started to use NCCN risk classification to list our patients on the waitlist, rather than the date of consult. As a practice, we also did not have to furlough or lay-off any employees, as all team 
Fig. 5 Inventory statistics of all Adventhealth facilities in Central Florida Division during the COVID-19 epidemic shutdown
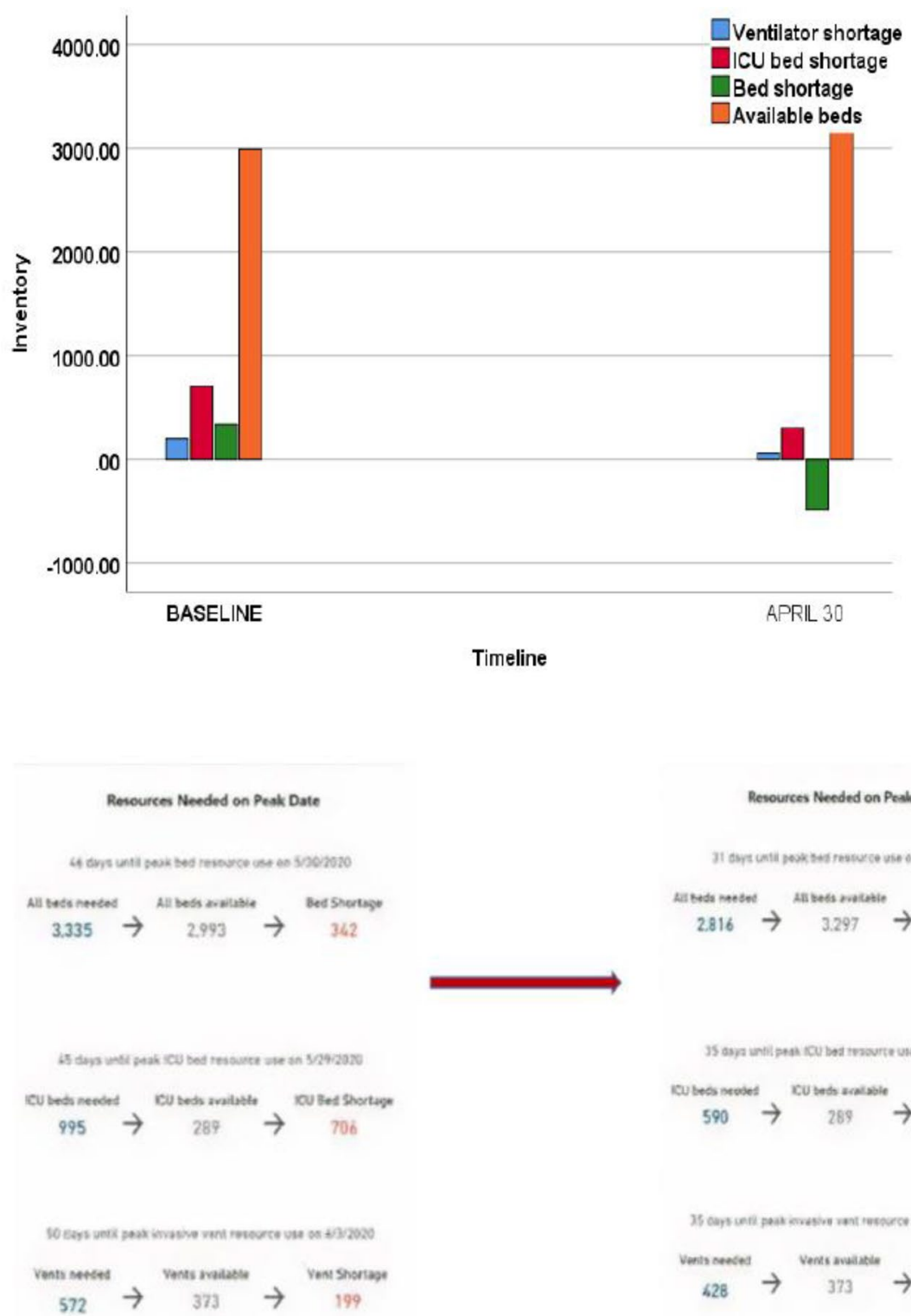

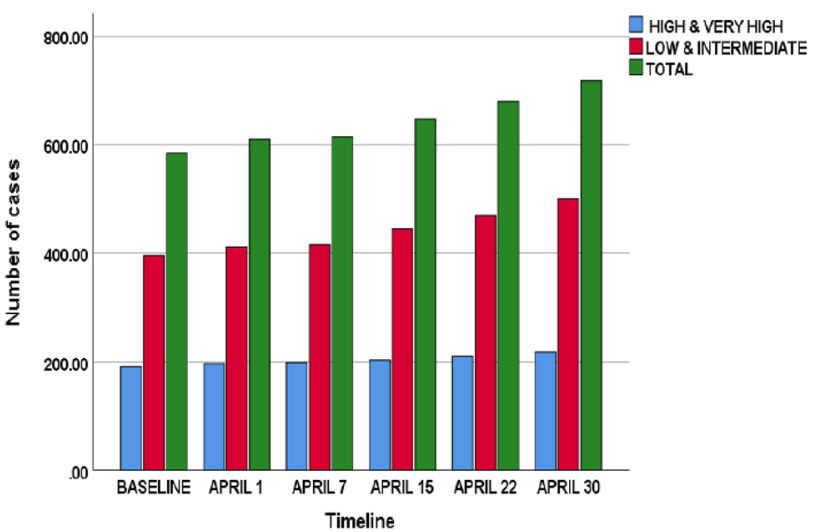

Fig. 6 Number of cases based on NCCN risk stratification each week members were occupied in triaging, planning, and contacting our waitlisted patients. All our patients were updated weekly about the situation, and this communication made them many comfortable, which in turn made them more compliant and satisfied.

\section{Weakness}

The whole COVID-19 situation has been overwhelming to our practice due to the delay it has caused. With a delay of 1 month, about 130 patients who could have been operated on were delayed. We are now operating, performing 8 prostatectomies a day, 5 days a week, which could ultimately lead to burnout of our team members. There is also a possibility that we would be operating on large volume disease 
Table 1 COVID-19 Risk stratification for elective surgical procedures

\begin{tabular}{|c|c|c|}
\hline Morbidty & Weight & $\begin{array}{l}\text { International } \\
\text { Consensus Score } \\
(\%)\end{array}$ \\
\hline
\end{tabular}

\begin{tabular}{lll}
\hline Age $>$ 50 & 1 & \\
Cardiac disease & 1 & 97 \\
Diabetes-no organ failure & 1 & 97 \\
$\quad$ With organ failure & 2 & 97 \\
Chronic lung disease & 3 & 97 \\
Heavy smoker & 2 & 83 \\
Liver disease & 1 & \\
CKD & 2 & 97 \\
Immunodeficiency & 3 & 97 \\
Steroid treatment & 1 & 88 \\
Autoimmune disorders & 1 & 83 \\
Ongoing chemotherapy & 2 & 91 \\
Obesity BMI $>$ 35 & 1 & 80 \\
\hline
\end{tabular}

Suggested risk stratification: two or $<2=$ low risk; $2-4=$ intermediate risk (needs icu); $>4=$ high risk (no surgery)

which could lead to longer OR time and more positive surgical margins [9].

\section{Opportunities}

Our approach was multi-disciplinary, involving Urologist, Medical Oncologist, and Radiation Oncologist. Surgery was determined to be the safer option when compared to radiation therapy, as the radiation oncologists here used the RADS approach which recommended deferring patients for radiotherapy as multiple visits could potentially increase the chance of contracting the disease [10]. We had a risk management team that included statisticians and a risk management company. The adoption of technology, such as videoconferencing, was a major change in our practice. Also, our institution is an academic center, and this temporary decrease in operative time increased our academic activities especially concerning COVID-19. Our faculty and fellows participated in many web seminars which were more convenient than physically traveling to actual meetings. Perhaps this will be the future of educational opportunities?

\section{Threats}

It could be stated that our approach could potentially lead to the progression of the disease, and one might argue that the COVID-19 situation is not very concerning and the need for suspending our surgeries is minimal. The process of waiting created more anxiety among patients, especially the HR and VHR group. Also, there is the risk of progression in intermediate/low-risk patients who could have undiagnosed high-risk disease. Challenges remain in procuring PPE due to global demand and lack of supply. The COVID-19 situation is not entirely under control, especially due to the lack of vaccines and treatment drugs. This could potentially lead to an increased risk of infection, both to our patients who are being operated on and also our staff. The state of Florida recently opened for all businesses which could potentially cause a surge in cases. This could once again place a burden on the healthcare system. Finally, as a practice, there is a potential economic effect caused by the loss of patients due to longer wait times.

\section{Discussion}

The COVID-19 pandemic is affecting all over the world. It has been a massive burden on the health care systems and has used up resources normally used to treat patients with chronic illness. Our center is a high-volume urologic cancer center managing a very high volume of prostate cancer patients. This paper is a description of changes to our practice in caring for patients with prostate cancer amidst the peak of the COVID-19 pandemic. Though we are not free from this pandemic, the viral curves in the state of Florida are beginning to flatten. With flat viral curves and low viral impact, relative to highly affected areas like Italy and New York, a reversal of stay at home order occurred on May 1st.

Prostate cancer management is based on the prostate cancer risk category. Even as little as a 3-month delay in the treatment of prostate cancer, especially HR and VHR disease, has potential long-term implications [11]. During the COVID-19 pandemic, the management of prostate cancer was deferred in areas of the high incidence of COVID-19, especially in Italy and New York. For example, in Papa Giovanni XXIII Hospital in Lombardy, where the COVID-19 incidence was high, all of the urologic procedures were suspended on the 19th of March due to the following reasons: (I) lack of anaesthesiologists, (II) use of beds and wards by COVID-19 patients, (III) diversion of urology staff to care for COVID-19 patients and (IV) risk of infecting nonCOVID-19 patients with the virus [12]. Assuming that this scenario could play out in our area as well, and as a response to the initial estimate, we suspended all elective procedures thus preserving our resources, namely PPE and hospital beds.

Our administration felt these steps were necessary, because there was an increase in the global demand of PPE, which ultimately stressed our PPE supply chains. These conditions made it hard for our hospital system to procure the needed PPE estimated by our pandemic response models. Once all of the nonemergent procedures were postponed, all of the patients were triaged into groups based on NCCN risk stratification. VHR and HR patients will be given priority upon the rescheduling of surgeries. Simultaneously, 
all of our patients were subcategorized by the COVID-19 risk category developed using a DELPHI consensus, created by a group of experts from all over the world. The use of video and phone patient visits was also a major change in our practice.

Throughout April, we monitored the situation closely, looking at the various models and the data from our reference centers. Though the situation looked unfavorable initially, the mitigation strategies implemented by the Governor of Florida reduced the impact of COVID-19 in this state. The basic requirements we used to determine whether or not it is safe to restart surgical procedures is the availability of N95 masks for each member of the surgical team, along with facial shields and shoe covers. This is in addition to regular surgical gloves. Surgical procedures resumed on the 4th of May in our hospital system. and was possible, because we managed our resources well during April. Proper management of resources made our hospital administration confident that we had adequate resources to tackle the new peak, which is predicted to be in mid-May. The state of Florida re-opened on May 1st due to stable COVID-19 predictive curves. Our SWOT analysis revealed our internal strengths and inherent weakness, relevant to the pandemic. According to the CDC, the threat posed by COVID-19 is still very real [13]. Currently, we are not sure if our approach is the right one, but at this point, it is probably the best we have available. Ultimately, our cancer patients are the ones who bare the brunt of our decisions. It is for this reason that we can not treat our response to situations like the COVID-19 pandemic lightly.

\section{Conclusion}

A pandemic caused by a novel virus-like COVID-19 is an overwhelming public health crisis. A graded and a guarded response to this type of situation is crucial in managing patients in a large practice such as ours. This is especially important when it comes to dealing with the treatment of cancer patients.

Funding Not applicable.

\section{Compliance with ethical standards}

Conflict of interest The authors declare that they have no conflict of interest.

Ethical approval All procedures followed were in accordance with the ethical standards of the responsible committee on human experimenta- tion (institutional and national) and with the Helsinki Declaration of 1975, as revised in 2000 .

\section{References}

1. Marandino L, Di Maio M, Procopio G, Cinieri S, Beretta GD, Necchi A (2020) The shifting landscape of genitourinary oncology during the COVID-19 pandemic and how Italian oncologists reacted: results from a national survey. Eur Urol 0

2. Seetharam Bhat KR, Moschovas MC, Onol FF, Sandri M, Rogers $\mathrm{T}$, Roof $\mathrm{S}$ et al (2020) Trends in clinical and oncological outcomes of robot-assisted radical prostatectomy before and after the 2012 US Preventive Services Task Force recommendation against PSA screening: a decade of experience. BJU Int [Internet]. https://doi. wiley.com/10.1111/bju.15051. Accessed 5 May 2020

3. Banerji JS, Wolff EM, Massman JD, Odem-Davis K, Porter CR, Corman JM (2015) Prostate needle biopsy outcomes in the era of the U.S. preventive services task force recommendation against psa-based screening. J Urol [Internet]. https://www.sciencedirect. com/science/article/pii/S0022534715045085. Accessed 30 Aug 2020

4. st_app (2020) Streamlit [Internet]. https://penn-chime.phl.io/. Accessed 5 May 2020

5. Responding to coronavirus: integrated nerve centerlMcKinsey [Internet]. https://www.mckinsey.com/business-functions/risk/ our-insights/responding-to-coronavirus-the-minimum-viabl e-nerve-center. Accessed 5 May 2020

6. SWOT analysis - Minnesota Dept. of Health [Internet]. https:// www.health.state.mn.us/communities/practice/resources/phqit oolbox/swot.html. Accessed 5 May 2020

7. Coronavirus (COVID-19) - google news [Internet]. https://news. google.com/covid19/map?hl=en-US\&gl=US\&ceid=US: en. Accessed 5 May 2020

8. Ma BL, Yao L, Fan Y, Wang Y, Meng YS, Zhang Q et al (2019) Short-term benefit of neoadjuvant hormone therapy in patients with localized high-risk or limited progressive prostate cancer. Cancer Manag Res 11:4143-4151

9. Salkini M (2020) The role of robot-assisted radical prostatectomy in high-risk organ-confined prostate cancer. Urol Ann 12:1-3

10. Zaorsky NG, Yu JB, McBride SM, Dess RT, Jackson WC, Mahal BA et al (2020) Prostate cancer radiation therapy recommendations in response to COVID-19. Adv Radiat Oncol. https://doi. org/10.1016/j.adro.2020.03.010

11. Wallis CJ, Novara G, Marandino L, Bex A, Kamat AM, Jeffrey Karnes R, et al. Risks from deferring treatment for genitourinary cancers: a collaborative review to aid triage and management during the COVID-19 pandemic. Affiliation: Italy submitting corresponding author: post-acceptance corresponding author

12. Naspro R, Da Pozzo LF (2020) Urology in the time of corona. Nat Rev Urol 17:251-253

13. Situation summarylCDC [Internet]. https://www.cdc.gov/coron avirus/2019-ncov/cases-updates/summary.html. Accessed 5 May 2020

Publisher's Note Springer Nature remains neutral with regard to jurisdictional claims in published maps and institutional affiliations. 